\title{
THE DIFFRACTION OF TRANSIENT ELECTRO- MAGNETIC WAVES BY A WEDGE
}

\author{
By A. C. BUTCHER and J. S. LOWNDES \\ (Received 18th July 1957)
}

\section{Introduction}

Much of the work on the theory of diffraction by an infinite wedge has been for cases of harmonic time-dependence. Oberhettinger (1) obtained an expression for the Green's function of the wave equation in the two dimensional case of a line source of oscillating current parallel to the edge of a wedge with perfectly conducting walls. Solutions of the time-dependent wave equation have been obtained by Keller and Blank (2), Kay (3) and more recently by Turner (4) who considered the diffraction of a cylindrical pulse by a half plane.

In this paper, using the methods of integral transforms, expressions are obtained for the field components due to a line source of current, whose density is some function of time, lying parallel to the edge of an infinite wedge with perfectly conducting walls. The general expressions are used to deduce the field components due to a step-function current source when the displacement currents inside the wedge are neglected. A second form for the field components is derived from these results which enables an analysis to be made of the field within the wedge into image terms and additional "perturbation" terms.

\section{Statement of the Problem}

An infinitely long line source of uniformly distributed current, whose density is a function of time alone, lies parallel to the edge of a wedge with perfectly conducting walls in a region of conductivity $\sigma$, dielectric constant $\epsilon$ and permeability $\mu$. The problem to be considered is referred to the cylindrical co-ordinate system $(r, \theta, z)$ where the tip of the wedge is co-incident with the $z$ axis.

Let $\alpha$ be the angle of the wedge and the line source pass through

$$
\left(r_{0}, \theta_{0}\right), 0 \leqslant \theta_{0} \leqslant a \text {. }
$$

Since the problem is two dimensional, Maxwell's equations (in m.k.s. units) for the region within the wedge are,

$$
\begin{gathered}
\frac{1}{r} \frac{\partial}{\partial r}\left(r H_{\theta}\right)-\frac{1}{r} \frac{\partial H_{r}}{\partial \theta}=\left(\sigma+\epsilon \frac{\partial}{\partial t}\right) E_{z}+M(t) \frac{\delta\left(r-r_{0}\right) \delta\left(\theta-\theta_{0}\right)}{r}, \\
\frac{1}{r} \frac{\partial E_{z}}{\partial \theta}=-\mu \frac{\partial H_{r}}{\partial t}, \frac{\partial E_{z}}{\partial r}=\mu \frac{\partial H_{\theta}}{\partial t}
\end{gathered}
$$

where

$$
M(t) \frac{\delta\left(r-r_{0}\right) \delta\left(\theta-\theta_{0}\right)}{r}
$$


represents a current density corresponding to a line current passing through $\left(r_{0}, \theta_{0}\right) . M(t)$ is a function of time alone and $\delta(x)$ denotes the Dirac delta function.

The boundary conditions to be applied are,

$$
\left.\begin{array}{l}
E_{z}=0 \text { on } \theta=0, a, \\
\mathrm{H}_{r}=H_{\theta}=E_{z}=0 \text { at } t=0 \text { and as } r \rightarrow \infty .
\end{array}\right\}
$$

\section{Solution of the Problem}

\subsection{First form of the solution}

Applying Laplace and finite Fourier transforms to equations (1), we get,

$$
\left.\begin{array}{c}
\frac{1}{r} \frac{d}{d r}\left(r H_{\theta}\right)+\frac{1}{r}\left(\frac{n \pi}{a}\right) A_{r}=(\sigma+\epsilon s) \bar{E}_{z}+m(s) \sin \frac{n \pi \theta_{0}}{a} \frac{\delta\left(r-r_{0}\right)}{r}, \\
\frac{1}{r}\left(\frac{n \pi}{a}\right) \bar{E}_{z}=-\mu s H_{r}, \frac{d \bar{E}_{z}}{d r}=\mu s H_{\theta}
\end{array}\right\}
$$

where

$$
\begin{aligned}
& \bar{E}_{z}(r, n, s)=\int_{0}^{a} \sin \frac{n \pi \theta}{a} d \theta \int_{0}^{\infty} E_{z}(r, \theta, t) e^{-s t} d t \\
& \vec{H}_{r}(r, n, s)=\int_{0}^{a} \cos \frac{n \pi \theta}{a} d \theta \int_{0}^{\infty} H_{r}(r, \theta, t) e^{-s t} d t, \\
& \hat{H}_{\theta}(r, n, s)=\int_{0}^{a} \sin \frac{n \pi \theta}{a} d \theta \int_{0}^{\infty} H_{\theta}(r, \theta, t) e^{-s t} d t,
\end{aligned}
$$

and $m(s)$ is the Laplace transform of $M(t)$.

Eliminating $\vec{H}_{r}, \vec{H}_{\theta}$ between equations (3) we find,

$$
\frac{1}{r} \frac{d}{d r}\left(r \frac{d \bar{E}_{z}}{d r}\right)-\left[k^{2}+\left(\frac{n \pi}{a r}\right)^{2}\right] \bar{E}_{z}=\mu s m(s) \frac{\delta\left(r-r_{0}\right)}{r} \sin \frac{n \pi \theta_{0}}{a},
$$

where $k^{2}=\mu s(\sigma+\epsilon s)$.

The solution of (4) is

$$
\bar{E}_{z}=-\mu s m(s) \sin \frac{n \pi \theta_{0}}{a} I_{n \pi / a}(k r) K_{n \pi / a}\left(k r_{0}\right), r<r_{0} .
$$

When $r_{0}<r$ the position of $r$ and $r_{0}$ are interchanged.

Inverting equation (5) with respect to the Laplace and Fourier transforms we have

$$
E_{z}=\frac{i \mu}{\pi \alpha} \sum_{n=1}^{\infty} \sin \frac{n \pi \theta_{0}}{a} \sin \frac{n \pi \theta}{a} \int_{\gamma-i \infty}^{\gamma+i \infty} s m(s) I_{n \pi / a}(k r) K_{n \pi / a}\left(k r_{0}\right) s t d s, r<r_{0} .
$$

Henceforth it is assumed that displacement currents can be neglected, i.e., set $\epsilon=0$. This means that the transient response is valid for times $t \gg \epsilon / \sigma$. We now consider the particular case of a current source whose density is proportional to a step function of time, i.e., $M(t)=M H(t)$ where $H(t)$ is the Heaviside unit function. Therefore $m(s)=M s^{-1}$. 
Substituting for $m(s)$ in equation (6) and evaluating the integral by ((5), p. 284) we arrive at the following expression which is valid for all $r$,

where

$$
E_{z}=-\mu M \exp \left[-\frac{\mu \sigma}{4 t}\left(r^{2}+r_{0}^{2}\right)\right] \frac{F(t)}{t}
$$

$$
F(t)=\frac{1}{\alpha} \sum_{n=1}^{\infty} \sin \frac{n \pi \theta_{0}}{a} \sin \frac{n \pi \theta}{a} I_{n \pi / a}\left(\beta_{/ t}\right), \beta=\frac{1}{2} \mu \sigma r r_{0} .
$$

Therefore from equations (7) and (1)

$$
\begin{aligned}
& H_{r}=\frac{M}{r} \int_{0}^{t} \frac{\partial}{\partial \theta} \exp \left[-\frac{\mu \sigma}{4 \tau}\left(r^{2}+r_{0}^{2}\right)\right] F(\tau) \frac{d \tau}{\tau}, \ldots \\
& H_{\theta}=-M \int_{0}^{t} \frac{\partial}{\partial r} \exp \left[-\frac{\mu \sigma}{4 \tau}\left(r^{2}+r_{0}^{2}\right)\right] F(\tau) \frac{d \tau}{\tau} .
\end{aligned}
$$

Equations (7), (8), (9) and (10) constitute the first form of the solution.

The series $F(t)$ is identical with that obtained by Carslaw and Jaeger $((6)$, p. 313) in an expression for the temperature due to an instantaneous point source situated inside an infinite wedge whose walls are maintained at zero temperature. This occurs because from equation (6) onwards we are, after putting $\epsilon=0$, solving essentially a heat conduction equation.

\subsection{Transformation of $\boldsymbol{F}(\boldsymbol{t})$}

We now give another form for the function $F(t)$ which will enable us to investigate further the field components.

We have from ((7), p. 181),

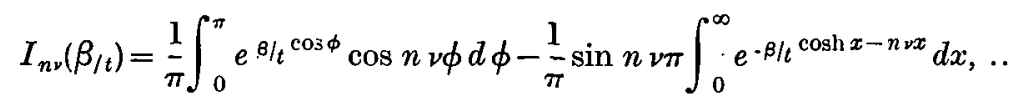

where $\pi_{/ a}=\nu=l+\lambda, l$ a positive integer and $\lambda$ such that $0 \leqslant \lambda<1$.

Substituting from equation (11) in equation (8) and summing Fourier and geometric series we have,

$$
F(t)=A+B+C,
$$

where

$$
\begin{aligned}
& A=\frac{1}{4 \pi} \sum_{m=0}^{l-1}\left\{f\left(\xi_{m}+m a\right)-f\left(\eta_{m}+m \alpha\right)\right\}, \\
& f(\zeta)=\exp \left[\beta_{/ t} \cos \zeta\right] \\
& \xi_{m}=\left|\theta-\theta_{0}\right|, m \text { even, } \eta_{m}=a-\left|\theta+\theta_{0}-\alpha\right|, m \text { even, } \\
& =a-\left|\theta-\theta_{0}\right|, m \text { odd, }=\left|\theta+\theta_{0}-\alpha\right|, m \text { odd. } \\
& B=\frac{1}{4 \pi}\left\{g\left(\xi_{l}\right)-g\left(\eta_{l}\right)\right\} \text {, } \\
& g(\zeta)=0, \quad \lambda a<\zeta<a, \\
& =\frac{1}{2} e^{-\beta / t}, \quad \zeta=\lambda \alpha, \\
& =\exp \left[-\beta_{/ t} \cos (\lambda \alpha-\zeta)\right]=f(\zeta+l \alpha), 0<\zeta<\lambda a \text {. }
\end{aligned}
$$




$$
\begin{gathered}
C=\frac{1}{8 \pi^{2}} \sum_{i=1}^{4}(-1)^{i} \int_{0}^{\infty} \frac{\exp \left[-\beta_{/ t} \cosh x_{/ v}\right] \sin \delta_{i}}{\cosh x-\cos \delta_{i}} d x, \\
\delta_{1}=\nu\left(\theta+\theta_{0}-\pi\right), \delta_{3}=\nu\left(\theta-\theta_{0}+\pi\right) \\
\delta_{2}=\nu\left(\theta+\theta_{0}+\pi\right), \delta_{4}=\nu\left(\theta-\theta_{0}-\pi\right) .
\end{gathered}
$$

TABLE I. $l$ Even

\begin{tabular}{|c|c|c|}
\hline $0<\theta_{0}<\delta$ & $g\left(\xi_{l}\right)$ & $g\left(\eta_{l}\right)$ \\
\hline $0<\theta<\lambda a-\theta_{0}$ & $f\left(\xi_{l}+l a\right)$ & $f\left(\eta_{l}+l a\right)$ \\
$\lambda a-\theta_{0}<\theta<\lambda a+\theta_{0}$ & $f\left(\xi_{l}+l a\right)$ & 0 \\
$\lambda a+\theta_{0}<\theta<a$ & 0 & 0 \\
\hline$\delta<\theta_{0}<a-\delta, \lambda<\frac{1}{2}$ & & \\
$0<\theta<\theta_{0}-\delta$ & & \\
$\theta_{0}-\delta<\theta<\theta_{0}+\delta$ & 0 & 0 \\
$\theta_{0}+\delta<\theta<a$ & $f\left(\xi_{l}+l a\right)$ & 0 \\
\hline$\delta<\theta_{0}<a-\delta, \lambda>\frac{1}{2}$ & 0 & 0 \\
\hline $0<\theta<a-\delta-\theta_{0}$ & & \\
$a-\theta_{0}-\delta<\theta<a-\theta_{0}+\delta$ & $f\left(\xi_{l}+l a\right)$ & $f\left(\eta_{l}+l a\right)$ \\
$a-\theta_{0}+\delta<\theta<a$ & 0 \\
\hline$a-\delta<\theta_{0}<a$ & $f\left(\xi_{l}+l a\right)$ & $f\left(\eta_{l}+l a\right)$ \\
\hline $0<\theta<\theta_{0}-\lambda a$ & & \\
$\theta_{0}-\lambda a<\theta<2 a-\lambda \alpha-\theta_{0}$ & $f\left(\xi_{l}+l a\right)$ & 0 \\
$2 a-\lambda \alpha-\theta_{0}<\theta<a$ & $f\left(\xi_{l}+l a\right)$ & $f\left(\eta_{l}+l a\right)$ \\
\hline
\end{tabular}

\subsection{Analysis of $B$ and $C$}

The definition of the function $g$ given in $\$ 3.2$ implies the existence of regions at the boundaries of which there are discontinuities in $B$.

Three cases of the angular position of the source have to be distinguished. These are

$$
0<\theta_{0}<\delta, \delta<\theta_{0}<\alpha-\delta, a-\delta<\theta_{0}<\alpha
$$

where

$$
\begin{aligned}
\delta & =\lambda \alpha, \lambda<\frac{1}{2}, \\
& =a-\lambda \alpha, \lambda>\frac{1}{2} .
\end{aligned}
$$

Naturally for $\lambda=\frac{1}{2}$ only two cases need to be distinguished. 
In each of these cases the form of $g\left(\xi_{l}\right)$ and $g\left(\eta_{l}\right)$ corresponding to the resulting " $\theta$ regions" are given in tables I and II.

Since $A$ is continuous and $B$ is discontinuous there must be compensating discontinuities in $C$. From tables $\mathrm{I}$ and $\mathrm{II}$ it is plain that on passing over

TABLE II. $l$ ODD

\begin{tabular}{|c|c|c|}
\hline $0<\theta_{0}<\delta$ & $g\left(\xi_{l}\right)$ & $g\left(\eta_{l}\right)$ \\
\hline $\begin{array}{l}0<\theta<a-\lambda a-\theta_{0} \\
a-\lambda \alpha-\theta_{0}<\theta<a-\lambda \alpha+\theta_{0} \\
a-\lambda \alpha+\theta_{0}<\theta<a\end{array}$ & $\begin{array}{c}0 \\
0 \\
f\left(\xi_{l}+l a\right)\end{array}$ & $\begin{array}{c}0 \\
f\left(\eta_{l}+l a\right) \\
f\left(\eta_{l}+l a\right)\end{array}$ \\
\hline \multicolumn{3}{|l|}{$\delta<\theta_{0}<\alpha-\delta, \lambda<\frac{1}{2}$} \\
\hline $\begin{array}{l}\quad 0<\theta<a-\theta_{0}-\delta \\
a-\theta_{0}-\delta<\theta<a-\theta_{0}+\delta \\
a-\theta_{0}+\delta<\theta<a\end{array}$ & $\begin{array}{l}0 \\
0 \\
0\end{array}$ & $\begin{array}{c}0 \\
f\left(\eta_{l}+l a\right) \\
0\end{array}$ \\
\hline \multicolumn{3}{|l|}{$\delta<\theta_{0}<a-\delta, \lambda>\frac{1}{2}$} \\
\hline $\begin{aligned} 0 & <\theta<\theta_{0}-\delta \\
\theta_{0}-\delta & <\theta<\theta_{0}+\delta \\
\theta_{0}+\delta & <\theta<a\end{aligned}$ & $\begin{array}{c}f\left(\xi_{l}+l a\right) \\
0 \\
f\left(\xi_{l}+l a\right)\end{array}$ & $\begin{array}{l}f\left(\eta_{l}+l a\right) \\
f\left(\eta_{l}+l a\right) \\
f\left(\eta_{l}+l a\right)\end{array}$ \\
\hline \multicolumn{3}{|l|}{$a-\delta<\theta_{0}<a$} \\
\hline $\begin{array}{c}0<\theta<\theta_{0}-a+\lambda a \\
\theta_{0}-a+\lambda \alpha<\theta<a+\lambda a-\theta_{0} \\
a+\lambda a-\theta_{0}<\theta<a\end{array}$ & $\begin{array}{c}f\left(\xi_{l}+l a\right) \\
0 \\
0\end{array}$ & $\begin{array}{c}f\left(\eta_{l}+l a\right) \\
f\left(\eta_{l}+l a\right) \\
0\end{array}$ \\
\hline
\end{tabular}

the boundaries one of the $\delta_{i}$ 's passes through an integral multiple of $2 \pi$. Therefore we need to consider the behaviour of

$$
K\left(\delta_{i}\right)=\int_{0}^{\infty} \frac{\exp \left[-\beta_{/ t} \cosh x / v\right] \sin \delta_{i}}{\cosh x-\cos \delta_{i}} d x
$$

as $\delta_{i}$ crosses the origin in a positive direction.

If we introduce two bounded functions $h(x), H(x)$ defined by

$$
\begin{gathered}
h(x)=h_{r}, H(x)=H_{r}, x_{r} \leqslant x \leqslant x_{r+1} \\
0=x_{0}<x_{1}<\ldots<x_{r}<x_{r+1}<\ldots,
\end{gathered}
$$

and such that $h(x)<\exp \left[-\beta_{/ t} \cosh x_{/ v}\right]<H(x)$ all $x$, we have

$$
2 \sum_{r=0}^{\infty} h_{r} \tan ^{-1} X_{r}<K\left(\delta_{i}\right)<2 \sum_{r=0}^{\infty} H_{r} \tan ^{-1} X_{r}
$$


where

$$
X_{r}=\frac{\cot \frac{\delta_{i}}{2}\left(\tanh \frac{x_{r+1}}{2}-\tanh \frac{x_{r}}{2}\right)}{1+\cot ^{2} \frac{\delta_{i}}{2} \tanh \frac{x_{r+1}}{2} \tanh \frac{x_{r}}{2}}
$$

Therefore

$$
\pi h_{0}<\lim _{\delta_{i} \rightarrow+0} K\left(\delta_{i}\right)<\pi H_{0} .
$$

Since the only restriction on $h_{0}, H_{0}$ is that they be bounded and $h_{0}<e^{-\beta_{t}}<H_{0}$ we have

similarly

and

$$
\left.\begin{array}{c}
\lim _{\delta_{i \rightarrow+0}} K\left(\delta_{i}\right)=\pi e^{-\beta / t} \\
\lim _{\delta_{i \rightarrow-0}} K\left(\delta_{i}\right)=-\pi e^{-\beta_{/ t}} \\
K(0)=0 .
\end{array}\right\}
$$

\begin{tabular}{|c|c|c|c|c|}
\hline & $\theta_{0}$ Region & $\begin{array}{l}\text { Number of ' } Q \text { ' } \\
\text { Virtual Images }\end{array}$ & $\theta_{0}$ Region & $\begin{array}{l}\text { Number of ' } R \text { ' } \\
\text { Virtual Images }\end{array}$ \\
\hline$\lambda<\frac{1}{2}$ & $\begin{array}{l}0<\theta_{0}<a-2 \lambda \alpha \\
a-2 \lambda \alpha<\theta_{0}<\alpha\end{array}$ & $\begin{array}{c}2 l-1 \\
2 l\end{array}$ & $\begin{array}{l}0<\theta_{0}<2 \lambda a \\
2 \lambda \alpha<\theta_{0}<\alpha\end{array}$ & $\begin{array}{c}2 l \\
2 l-1\end{array}$ \\
\hline$\lambda>\frac{1}{2}$ & $\begin{array}{l}0<\theta_{0}<2 a-2 \lambda a \\
2 a-2 \lambda a<\theta_{0}<a\end{array}$ & $\begin{array}{c}2 l \\
2 l+1\end{array}$ & $\begin{array}{l}0<\theta_{0}<2 \lambda a-a \\
2 \lambda a-a<\theta_{0}<a\end{array}$ & $\begin{array}{c}2 l+1 \\
2 l\end{array}$ \\
\hline
\end{tabular}

TABLE III

This exactly compensates for the discontinuities in $B$.

Oberhettinger (1) has shown for $a>\pi$ that the boundaries at which the analytic form of the solution changes can be connected with the visibility of the source and its images from the observation point, with reference to the walls of the wedge. It will now be shown that this is also true for $a \leqslant \pi$.

Denoting by $Q_{a}^{n}, Q_{0}^{n}$ the angular coordinates of the $n$th image in the walls $\theta=\alpha, \theta=0$ respectively, on reflection first in $\theta=a$, and by $R_{0}^{n}, R_{a}^{n}$ the angular coordinates of the $n$th image in the walls $\theta=0, \theta=a$ respectively, on reflection first in $\theta=0$; we get

$$
\begin{array}{ll}
Q_{a}^{n}=2 n a-\theta_{0}, & R_{0}^{n}=-2(n-1) a-\theta_{0} \\
Q_{0}^{n}=-2 n a+\theta_{0}, & R_{a}^{n}=2 n a+\theta_{0}
\end{array}
$$

The limitation on the total number of images for $a \leqslant \pi$ arising from the occurrence of real images is summarised in table III. 
We now define a " complementary" region to the wedge by $\pi<\theta<\pi+a$.

Images falling in the complementary region either become visible or cease to be visible on crossing the line produced connecting the image to the edge of the wedge, unless they can be regarded as images in both walls simultaneously which only occurs when $a$ is a sub-multiple of $\pi$.

By considering the angular coordinates of images falling in the complementary region we obtain boundaries which are summarised in table IV.

On analysis into $\lambda<\frac{1}{2}, \lambda>\frac{1}{2}$ the boundaries given in table IV agree with those indicated in tables $I$ and II, i.e., the regions of tables I and II coincide with the regions of visibility of the source and its images.

Table IV

\begin{tabular}{|l|c|c|}
\hline \multirow{2}{*}{ Source Regions } & \multicolumn{2}{|c|}{ ' $\theta$ ' Boundaries } \\
\cline { 2 - 3 } & $l$ Even & $l$ Odd \\
\hline $0<\theta_{0}<\lambda a$ & $\lambda a-\theta_{0}$ & $a-\lambda a+\theta_{0}$ \\
$\lambda a<\theta_{0}<a$ & $\theta_{0}-\lambda a$ & $a+\lambda a-\theta_{0}$ \\
$0<\theta_{0}<a-\lambda a$ & $\lambda a+\theta_{0}$ & $a-\lambda a-\theta_{0}$ \\
$a-\lambda a<\theta_{0}<a$ & $2 a-\lambda a-\theta_{0}$ & $\theta_{0}+\lambda a-a$ \\
\hline
\end{tabular}

For $\pi<a<2 \pi, l=0$, and $\frac{1}{2} \leqslant \lambda<1$, the part $A$ does not exist, $\delta=\lambda a$ and the forms of $g\left(\xi_{l}\right), g\left(\eta_{l}\right)$ are given by table I. In this case the resulting " $\theta$ regions" reduce to those obtained by Oberhettinger (1) who in another paper (8) has considered integrals of the type (16) for large and small $r$.

\subsection{Second form of the solution}

We now substitute for $F(t)$ from equation (12) in equations (7), (9) and (10). Denoting by $E_{z}^{A}, E_{z}^{B}, E_{z}^{C}$ the contribution to $E_{z}$ from parts $A, B$ and $C$ respectively and similarly for $H_{r}, H_{\theta}$ we find

\section{Contribution from part $A$}

$$
\begin{gathered}
E_{z}^{A}=-\frac{\mu M}{4 \pi t} \exp \left[-\frac{\mu \sigma}{4 t}\left(r^{2}+r_{0}^{2}\right)\right]_{m=0}^{l-1}\left\{f\left(\xi_{m}+m a\right)-f\left(\eta_{m}+m a\right)\right\}, \ldots \ldots \\
H_{r}^{A}=\frac{M r_{0}}{2 \pi} \exp \left[-\frac{\mu \sigma}{4 t}\left(r^{2}+r_{0}^{2}\right)\right]_{m=0}^{l-1}\left\{\frac{\left(\frac{\partial \xi_{m}}{\partial \theta}\right) \sin \left(\xi_{m}+m a\right) f\left(\xi_{m}+m a\right)}{2 r r_{0} \cos \left(\xi_{m}+m a\right)-r^{2}-r_{0}^{2}}\right. \\
\left.-\frac{\left(\frac{\partial \eta_{m}}{\partial \theta}\right) \sin \left(\eta_{m}+m a\right) f\left(\eta_{m}+m a\right)}{2 r r_{0} \cos \left(\eta_{m}+m a\right)-r^{2}-r_{0}^{2}}\right\}, \ldots \ldots \ldots
\end{gathered}
$$




$$
\begin{array}{r}
H_{\theta}^{A}=-\frac{M}{2 \pi} \exp \left[-\frac{\mu \sigma}{4 t}\left(r^{2}+r_{0}^{2}\right)\right] \sum_{m=0}^{l-1}\left\{\frac{\left[r-r_{0} \cos \left(\xi_{m}+m a\right)\right] f\left(\xi_{m}+m a\right)}{2 r r_{0} \cos \left(\xi_{m}+m a\right)-r^{2}-r_{0}^{2}}\right. \\
\left.-\frac{\left[r-r_{0} \cos \left(\eta_{m}+m a\right)\right] f\left(\eta_{m}+m a\right)}{2 r r_{0} \cos \left(\eta_{m}+m a\right)-r^{2}-r_{0}^{2}}\right\} \ldots \ldots \ldots .
\end{array}
$$

\section{Contribution from part $B$}

$$
\begin{aligned}
E_{z}^{B}=-\frac{\mu M}{4 \pi t} \exp [ & \left.-\frac{\mu \sigma}{4 t}\left(r^{2}+r_{0}^{2}\right)\right]\left\{g\left(\xi_{l}\right)-g\left(\eta_{l}\right)\right\}, \\
H_{r}^{B}=\frac{M r_{0}}{2 \pi} \exp \left[-\frac{\mu \sigma}{4 t}\left(r^{2}+r_{0}^{2}\right)\right] & \left\{\frac{\left(\frac{\partial \xi_{l}}{\partial \theta}\right) \sin \left(\xi_{l}+l a\right) g\left(\xi_{l}\right)}{2 r r_{0} \cos \left(\xi_{l}+l a\right)-r^{2}-r_{0}^{2}}\right. \\
& \left.-\frac{\left(\frac{\partial \eta_{l}}{\partial \theta}\right) \sin \left(\eta_{l}+l a\right) g\left(\eta_{l}\right)}{2 r r_{0} \cos \left(\eta_{l}+l a\right)-r^{2}-r_{0}^{2}}\right\} \\
H_{\theta}^{B}=-\frac{M}{2 \pi} \exp \left[-\frac{\mu \sigma}{4 t}\left(r^{2}+r_{0}^{2}\right)\right] & \left\{\frac{\left[r-r_{0} \cos \left(\xi_{l}+l a\right)\right] g\left(\xi_{l}\right)}{2 r r_{0} \cos \left(\xi_{l}+l a\right)-r^{2}-r_{0}^{2}}\right. \\
& \left.-\frac{\left[r-r_{0} \cos \left(\eta_{l}+l a\right)\right] g\left(\eta_{l}\right)}{2 r r_{0} \cos \left(\eta_{l}+l a\right)-r^{2}-r_{0}^{2}}\right\} .
\end{aligned}
$$

\section{Contribution from part $C$}

$$
\begin{aligned}
& E_{z}^{C}=-\frac{\mu M}{8 \pi a t} \exp \left[-\frac{\mu \sigma}{4 t}\left(r^{2}+r_{0}^{2}\right)\right] \sum_{i=1}^{4}(-1)^{i} \sin \delta_{i} \int_{0}^{\infty} \frac{\exp \left[-\beta_{/ t} \cosh x\right]}{\cosh \nu x-\cos \delta_{i}} d x, \\
& H_{r}^{C}=\frac{M}{8 r \alpha^{2}} \sum_{i=1}^{4}(-1)^{i} \int_{0}^{\infty}-E i\left(-\frac{\mu \sigma}{4 t}\left[r^{2}+r_{0}^{2}+2 r r_{0} \cosh x\right]\right) \\
& \times \frac{\cosh \nu x \cos \delta_{i}-1}{\left(\cosh \nu x-\cos \delta_{i}\right)^{2}} d x \\
& H_{\theta}^{C}=\frac{M}{4 \pi a} \exp \left[-\frac{\mu \sigma}{4 t}\left(r^{2}+r_{0}^{2}\right)\right] \underset{i=1}{\stackrel{4}{\sum}}(-1)^{i} \sin \delta_{i} \\
& \times \int_{0}^{\infty} \frac{\left(r+r_{0} \cosh x\right) \exp \left[-\beta_{/ t} \cosh x\right]}{\left(\cosh \nu x-\cos \delta_{i}\right)\left(r^{2}+r_{0}^{2}+2 r r_{0} \cosh x\right)} d x
\end{aligned}
$$

where

$$
-E i(-x)=\int_{x}^{\infty} e^{-u} \frac{d u}{u}
$$




\section{DIFFRACTION OF TRANSIENT ELECTRO-MAGNETIC WAVES 103}

\section{Acknowledgments}

We would like to thank Professor I. N. Sneddon and Mr B. Noble for helpful suggestions.

\section{REFERENCES}

(1) F. Oberhettinger, Comm. Pure and Appl. Math., 7 (1954), 551.

(2) J. Keller and A. Blank, Comm. Pure and Appl. Math., 4 (1951), 75.

(3) I. Kay, Comm. Pure and Appl. Math., 6 (1953), 419.

(4) R. D. Turner, Quart. Appl. Math., 14 (1956), 63.

(5) Erdelyi et al., Tables of Integral Transforms (McGraw-Hill, 1954).

(6) H. S. Carslaw and J. C. Jaeger, Conduction of Heat in Solids (Oxford, 1947).

(7) G. N. Watson, Theory of Bessel Functions (Cambridge, 1944).

(8) F. Oberhettinger, J. Math. Phys., 34 (1956), 245.

Armament Research and Development Establishment Fort Halstead

KENT 